\title{
Hybrid-automaton models for simulating systems with sliding motion: Still a challenge
}

Link to publication record in Manchester Research Explorer

\section{Citation for published version (APA):}

Navarro-López, E. M. (2009). Hybrid-automaton models for simulating systems with sliding motion: Still a challenge. In IFAC Proceedings Volumes (IFAC-PapersOnline)/IFAC Proc. Vol. (IFAC-PapersOnline) (Vol. 3, pp. 322-327). International Federation of Automatic Control (IFAC).

\section{Published in:}

IFAC Proceedings Volumes (IFAC-PapersOnline)|IFAC Proc. Vol. (IFAC-PapersOnline)

\section{Citing this paper}

Please note that where the full-text provided on Manchester Research Explorer is the Author Accepted Manuscript or Proof version this may differ from the final Published version. If citing, it is advised that you check and use the publisher's definitive version.

\section{General rights}

Copyright and moral rights for the publications made accessible in the Research Explorer are retained by the authors and/or other copyright owners and it is a condition of accessing publications that users recognise and abide by the legal requirements associated with these rights.

\section{Takedown policy}

If you believe that this document breaches copyright please refer to the University of Manchester's Takedown Procedures [http://man.ac.uk/04Y6Bo] or contact uml.scholarlycommunications@manchester.ac.uk providing relevant details, so we can investigate your claim.

\section{OPEN ACCESS}




\title{
Hybrid-automaton models for simulating systems with sliding motion: still a challenge
}

\author{
Eva M. Navarro-López \\ School of Computer Science, The University of Manchester, Oxford Road, \\ Kilburn Building, Manchester, M13 9PL, UK (e-mail: \\ eva.navarro@cs.man.ac.uk)
}

\begin{abstract}
There is a gap between the modelling and the simulation of discontinuous dynamical systems (DDS) exhibiting sliding-mode behaviour. Several challenges arise. Mainly, the definition of unique solutions on the discontinuity surfaces and at their crossings. There are also numerical issues in the detection and location of the trajectory entering and going out of the discontinuity surface, as well as when it remains on the discontinuity surface (chattering phenomenon). The aim of this paper is establishing a semantics for DDS so that a deterministic dynamical behaviour can be defined for simulation purposes. Particularly, a class of DDS is modelled by using two different hybrid automata. One of them is called the DDS hybrid automaton, proposed by the author previously. The other is obtained by modifying the DDS hybrid automaton, and is inspired in computer-simulation-oriented friction models. The use of a computational model is an elegant way for specifying the multiple transitions in DDS. The modelling framework is specially useful for specifying transitions when multiple discontinuity surfaces are present. A system with discontinuous friction and stick-slip oscillations is used to validate the models. In the example, it is concluded that discontinuous systems can be appropriately simulated by using hybrid-automaton-based models without the problem of chattering. On the other hand, the system dynamical behaviour can change depending on the step size of the numerical integration method and on the hybrid-automaton representation used. The hybrid models are simulated by means of Simulink/Stateflow ${ }^{\mathbb{R}}$ package.
\end{abstract}

Keywords: Hybrid systems; Discontinuous systems; Hybrid automata; Computational models; Friction; Simulation; Stateflow $\mathrm{R}$.

\section{MOTIVATION}

How to make completely deterministic, for simulation purposes, the dynamics of a discontinuous system exhibiting sliding motion? What about using hybrid models for this purpose? What are the dynamical implications of rewriting discontinuous dynamical systems as hybrid automata? What about the simulation of these hybrid automata? This paper attempts to answer these questions.

It is well-known that non-uniqueness or even non-existence of solutions may arise in discontinuous dynamical systems (DDS) when the trajectories cross or slide on the discontinuity surfaces. This has been extensively studied in systems with Coulomb friction and is generically referred to as the Painlavé paradox. The non-uniqueness of solutions, even for simple systems, can appear if the system dynamics and all the transitions are not appropriately specified Lötstedt (1981). A complete overview of the problem can be found in Brogliato (1999).

The problem of uniquely defining the solution in a DDS has been solved by means of different methods Filippov (1988); Utkin (1992). However, there are different challenges concerning the simulation and the numerical integration of these systems Acary and Brogliato (2008). There are two main issues. Firstly, maintaining the trajectory on the discontinuity surface once it has entered the surface, what is called the tracking error. Several numerical solutions have solved this problem and are closely related to avoid the chattering phenomenon Zhao and
Utkin (1996); Mosterman et al. (1999). Secondly, detecting and locating the trajectory crossing the discontinuity surface Park and Barton (1996); Zhang et al. (2008).

In the last decade, there has been an effort in proposing different semantics and computational-oriented frameworks for modelling systems exhibiting sliding-type behaviour. For example, object-oriented models Elmqvist et al. (1993); Mattsson (1996) or hybrid dynamic models Mosterman and Biswas (2000) are used for different applications.

Using a computational model is an elegant way in order to specify the transitions and event-triggered phenomena appearing in DDS's. In this paper, two different hybrid models are proposed for this purpose. The automaton-based framework is used Alur et al. (1993); Henzinger (1996). The basic hybrid automaton model is extracted from Johansson et al. (1999); Lygeros et al. (1999, 2003). It is very similar to the Hybrid State Model (HSM) proposed in Buss et al. (2002). The main difference between the HSM and the basic hybrid model used here, is that the HSM uses an equation-based representation, and the discontinuity surfaces are defined by means of switching functions instead of guard sets.

The two proposed hybrid automata model DDS's with one discontinuity surface. Systems with multiple discontinuity surfaces can be modelled by means of the composition of several hybrid automata Navarro-López (2009c). The first hybrid model, called the DDS hybrid automaton, was previously pro- 
posed by the author Navarro-López (2009c), and has three discrete locations. This model overcomes some problems encountered in the 3-discrete-states object-oriented model given in Mattsson (1996). The second hybrid model is a variation of the DDS hybrid automaton with five discrete locations. It is inspired in simulation-oriented models of discontinuous friction Karnopp (1985); Leine et al. (1998), and in the state-transition diagram of a friction model presented in Elmqvist et al. (1993). In comparison to Elmqvist et al. (1993), here, some guard and location domain sets have been changed so that the dynamics in each discrete location are uniquely defined.

The specification of DDS's given in this paper leads to a simulation algorithm. The events or discrete transitions are defined in order to clearly specify all the possible changes in the dynamics. As a consequence, the hybrid automata proposed can be translated to a program or to any other description language.

The simulation of the hybrid dynamical systems is carried out by means of the Simulink/Stateflow ${ }^{\circledR}$ toolbox of MATLAB ${ }^{\circledR}$ The MathWorks, Inc. (1999-2003). The translation between the Simulink/Stateflow models and the hybrid automata is far from being trivial Agrawal et al. (2004); Alur et al. (2008).

In order to validate the hybrid models, a system with discontinuous friction and different sliding-mode-related dynamics is considered. It is the torsional model of a conventional vertical oilwell drillstring of 2 degrees of freedom (DOF), which has been widely studied by the author, for instance, Navarro-López and Cortés (2007); Navarro-López and Licéaga-Castro (2009a); Navarro-López (2009b) and references therein.

\section{A HYBRID AUTOMATON FOR DISCONTINUOUS DYNAMICAL SYSTEMS}

The following general hybrid automaton Navarro-López (2009c) is used. It is based on the hybrid model given in Johansson et al. (1999); Lygeros et al. (1999, 2003).

Definition 1. A hybrid automaton with inputs and outputs is a collection

$$
H=(Q, E, \mathscr{X}, \Sigma, \mathscr{U}, O, \mathscr{Y}, \text { Dom, } \mathscr{F}, \text { Init }, G, R, h, r)
$$

where:

- $Q=\left\{q_{1}, q_{2}, \ldots, q_{\mathrm{N}}\right\}$ is a finite set of discrete states or locations.

- $E \subseteq Q \times Q$ is a finite set of edges called transitions or events.

- $\mathscr{X} \subseteq \mathbb{R}^{\mathrm{n}}$ is the continuous state space. $\mathscr{U} \subseteq \mathbb{R}^{\mathrm{m}}$ and $\mathscr{Y} \subseteq$ $\mathbb{R}^{\mathrm{m}}$ are the continuous input and output spaces, respectively. Moreover, $\mathscr{X}_{\mathrm{i}} \subseteq \mathscr{X}, \mathscr{U}_{\mathrm{i}} \subseteq \mathscr{U}$ and $\mathscr{Y}_{\mathrm{i}} \subseteq \mathscr{Y}$ are the continuous state, input and output spaces corresponding to each location $q_{\mathrm{i}}$. The state of $H$ is $(q, \mathbf{x}) \in Q \times \mathscr{X}$.

- $\Sigma=\left\{\sigma_{1}, \sigma_{2}, \ldots, \sigma_{\mathrm{M}}\right\}$ is a finite set of symbols labelling the edges and representing the discrete input events.

- $O=\left\{o_{1}, o_{2}, \ldots, o_{\mathrm{K}}\right\}$ is a finite set of symbols representing the discrete output events.

- Dom : $Q \rightarrow 2^{\mathscr{X} \times \mathscr{U}}$ is the location domain. It is a mapping from the locations $Q$ to the set of all subsets of $\mathscr{X} \times \mathscr{U}$, that is, Dom assigns a set of continuous states and inputs to each discrete state $q_{\mathrm{i}} \in Q$, thus, $\operatorname{Dom}\left(q_{\mathrm{i}}\right) \subset \mathscr{X} \times \mathscr{U}$.

- $\mathscr{F}=\left\{\mathbf{f}_{\mathrm{q}_{\mathrm{i}}}(\mathbf{x}, \mathbf{u}): q_{\mathrm{i}} \in Q\right\}$ is the collection of vector fields describing the continuous dynamics such that $f_{\mathrm{q}_{\mathrm{i}}}$ : $\mathscr{X} \times \mathscr{U} \rightarrow \mathscr{X}$. Each $\mathbf{f}_{\mathrm{q}_{\mathrm{i}}}(\mathbf{x}, \cdot)$ is assumed to be Lipschitz continuous on the location domain for $q_{\mathrm{i}}$ in order to ensure that the solution within $\mathscr{X}_{\mathrm{i}}$ exists and is unique.
- Init $\subseteq Q \times \mathscr{X}$ is a set of initial states.

- $G: E \rightarrow 2^{\mathscr{X}}$ is a guard set. Funtion $G$ assigns to each edge $e=\left(q_{\mathrm{i}}, q_{\mathrm{j}}\right) \in E$ a set of continuous states $(G(e) \subset \mathscr{X})$. Each guard set plays the role of an enabling condition in order to change the location.

- $R: E \times \mathscr{X} \times \mathscr{U} \rightarrow 2^{\mathscr{X}}$ is a reset map for the continuous states for each edge. It is assumed that $\forall e \in E, G(e) \neq \emptyset$ and $\forall \mathbf{x} \in G(e), R(e, \mathbf{x}, \mathbf{u}) \neq \emptyset$.

- $h: Q \times \mathscr{X} \times \mathscr{U} \rightarrow \mathscr{Y}$ is the continuous output mapping, there is one for each location.

- $r: Q \times \mathscr{X} \times \Sigma \times \mathscr{U} \rightarrow \mathscr{O}$ is the discrete output map, there is one for each location.

As long as the system is within location $q_{\mathrm{i}}$, the continuous state $\mathbf{x}$ must satisfy $\mathbf{x} \in \operatorname{Dom}\left(q_{\mathrm{i}}\right)$. The transition from a discrete state $q_{\mathrm{i}}$ to another $q_{\mathrm{j}}$ is enabled when the continuous state $\mathbf{x}$ reaches the guard $G\left(q_{\mathrm{i}}, q_{\mathrm{j}}\right) \subset \mathscr{X}$ of some edge $\left(q_{\mathrm{i}}, q_{\mathrm{j}}\right) \in E$. Then, the discrete state changes to $q_{\mathrm{j}}$ and at the same time, $\mathbf{x}$ is reset to the value specified by $R\left(q_{\mathrm{i}}, q_{\mathrm{j}}, \mathbf{x}, \mathbf{u}\right) \subset \mathscr{X} . H$ will be represented as a directed graph $(Q, E)$ with vertices $Q$ and edges $E$. For each vertex $q_{\mathrm{i}} \in Q$, a set of initial conditions, a vector field and a domain are given. In addition, a guard, a label and a reset function are associated with each edge, $e \in E$.

Now, a DDS with one discontinuity (or switching) surface is modelled as a hybrid automaton $H$. A state-dependent input control $\mathbf{u}(\mathbf{x})$ is used, consequently, the vector fields can be written as $\mathbf{f}_{\mathrm{q}_{\mathrm{i}}}(\mathbf{x})$, and,

$$
\dot{\mathbf{x}}=\left\{\begin{array}{l}
\mathbf{f}^{+}(\mathbf{x}) \text { if } \mathbf{x} \in S^{+} \\
\mathbf{f}^{-}(\mathbf{x}) \text { if } \mathbf{x} \in S^{-}
\end{array}\right.
$$

where $\mathbf{x} \in \mathscr{X} \subseteq \mathbb{R}^{\mathrm{n}}$ is the state vector, $\mathbf{f}^{+}$and $\mathbf{f}^{-}$are continuous and smooth, and $S^{+}=\{\mathbf{x} \in \mathscr{X}: s(\mathbf{x})>0\}, S^{-}=\{\mathbf{x} \in \mathscr{X}:$ $s(\mathbf{x})<0\}$, with $s$ a smooth scalar function with nonvanishing gradient. The discontinuity surface is $S=\{\mathbf{x} \in \mathscr{X}: s(\mathbf{x})=0\}$, and $\mathscr{X}=S^{+} \bigcup S^{-} \bigcup S$. On $S, \mathbf{f}^{+}(\mathbf{x})$ and $\mathbf{f}^{-}(\mathbf{x})$ do not agree.

The discontinuity surface is divided into two regions, the sliding set $S_{\mathrm{s}}$, which is closed, and the crossing set $S_{\mathrm{c}}$, which is open. Then $S=S_{\mathrm{S}} \cup S_{\mathrm{c}}$. $S_{\mathrm{S}}$ is the set where a sliding motion can take place, and $S_{\mathrm{c}}$ is the set of $S$ within which the trajectory crosses $S$ without sliding. The system dynamics on $S$ are $\dot{\mathbf{x}}=\mathbf{f}_{\mathrm{s}}(\mathbf{x})$, where $\mathbf{f}_{\mathrm{S}}$ is the equivalent dynamics Filippov (1988); Utkin (1992).

In this paper, the Utkin's equivalent control method is used Utkin (1992), which, as it is established in Zhao and Utkin (1996); Mosterman et al. (1999), gives better chatter-free simulation results for some cases. Then,

$$
\mathbf{f}_{\mathrm{s}}(\mathbf{x})=\frac{\mathbf{f}^{+}(\mathbf{x})+\mathbf{f}^{-}(\mathbf{x})}{2}+u_{\mathrm{eq}}(\mathbf{x}) \frac{\mathbf{f}^{-}(\mathbf{x})-\mathbf{f}^{+}(\mathbf{x})}{2}
$$

with $u_{\text {eq }}(\mathbf{x}) \in[-1,1]$ a scalar function playing the role of the equivalent control. From (2), and taking into account that $\mathbf{f}_{\mathrm{s}}$ must be tangential to $S$, one yields to,

$$
u_{\mathrm{eq}}(\mathbf{x})=-\frac{\left\langle\nabla s(\mathbf{x}), \mathbf{f}^{+}(\mathbf{x})\right\rangle+\left\langle\nabla s(\mathbf{x}), \mathbf{f}^{-}(\mathbf{x})\right\rangle}{\left\langle\nabla s(\mathbf{x}), \mathbf{f}^{-}(\mathbf{x})\right\rangle-\left\langle\nabla s(\mathbf{x}), \mathbf{f}^{+}(\mathbf{x})\right\rangle},
$$

where $\nabla s$ is the gradient of $s$ and $\langle\cdot, \cdot\rangle$ denotes the scalar product of vectors. The sliding set has the following form,

$$
S_{\mathrm{s}}:=\left\{\mathbf{x} \in S:-1 \leq u_{\mathrm{eq}}(\mathbf{x}) \leq 1\right\} .
$$

$S_{\mathrm{c}}$ is the complement set of $S_{\mathrm{s}}$ in $S$. It is assumed that there are no points on $S_{\mathrm{s}}$ at which both $f^{+}$and $f^{-}$are tangent to $S$. Furthermore, the sliding set is attractive for $\mathbf{x}$ such that $\left\langle\nabla s(\mathbf{x}), \mathbf{f}^{-}(\mathbf{x})\right\rangle-\left\langle\nabla s(\mathbf{x}), \mathbf{f}^{+}(\mathbf{x})\right\rangle>0$ and repulsive for $\mathbf{x}$ such that $\left\langle\nabla s(\mathbf{x}), \mathbf{f}^{-}(\mathbf{x})\right\rangle-\left\langle\nabla s(\mathbf{x}), \mathbf{f}^{+}(\mathbf{x})\right\rangle<0$ Kuznetsov (2003). 
Definition 2. Navarro-López (2009c) A DDS hybrid automaton with 3 discrete states $\left(H_{\mathrm{DDS}_{1}}\right)$ describing the dynamics of system (1) is a particular case of $H$ with,

- $Q=\left\{q_{1}, q_{2}, q_{3}\right\}=\left\{\right.$ slip $^{+}$, slip $^{-}$, stick $\}, \mathscr{X} \subseteq \mathbb{R}^{\mathrm{n}}$.

- $E=\left\{\left(q_{1}, q_{2}\right),\left(q_{1}, q_{3}\right),\left(q_{2}, q_{1}\right),\left(q_{2}, q_{3}\right),\left(q_{3}, q_{1}\right),\left(q_{3}, q_{2}\right)\right\}$.

- $\Sigma=\{a, b, c\}$, one edge label is assigned to each type of guard. For specification purposes, these discrete inputs can be considered redundant and unnecessary.

- $\operatorname{Dom}\left(q_{1}\right)=S^{+}, \operatorname{Dom}\left(q_{2}\right)=S^{-}, \operatorname{Dom}\left(q_{3}\right)=S$.

- $f_{\mathrm{q}_{1}}(\mathbf{x})=f^{+}(\mathbf{x}), f_{\mathrm{q}_{2}}(\mathbf{x})=f^{-}(\mathbf{x}), f_{\mathrm{q}_{3}}(\mathbf{x})=f_{\mathrm{s}}(\mathbf{x})$.

- Init $=Q \times \mathscr{X} / U_{\mathrm{s}}$, with $U_{\mathrm{s}}:=\left\{\mathbf{x} \in S_{\mathrm{s}}:\left\langle\nabla s(\mathbf{x}), \mathbf{f}^{-}(\mathbf{x})\right\rangle-\right.$ $\left.\left\langle\nabla s(\mathbf{x}), \mathbf{f}^{+}(\mathbf{x})\right\rangle<0\right\}$. Then, the problem of nonuniqueness of solutions starting at unstable sliding sets is avoided. Indeed, in most cases, solutions starting away from unstable sliding surfaces do not usually reach them.

- $G\left(q_{1}, q_{3}\right)=G\left(q_{2}, q_{3}\right)=S_{\mathrm{s}}, G\left(q_{1}, q_{2}\right)=G\left(q_{3}, q_{2}\right)=\{\mathbf{x} \in$ $\left.S: u_{\mathrm{eq}}(\mathbf{x})>1\right\}, G\left(q_{2}, q_{1}\right)=G\left(q_{3}, q_{1}\right)=\left\{\mathbf{x} \in S: u_{\mathrm{eq}}(\mathbf{x})<\right.$ $-1\}$.

- $R\left(q_{\mathrm{i}}, q_{\mathrm{j}}, \mathbf{x}\right)=\{\mathbf{x}\}, \forall i, j \in\{1,2,3\}$.

- $y=h\left(q_{1}, x\right)=h\left(q_{2}, x\right)=h\left(q_{3}, x\right)$ is the continuous output, which is the same for all the locations. No discrete outputs are considered.

A similar hybrid model is obtained in Sedghi et al. (2002); Sedghi (2003). The difference with this work is that the hybrid framework used in Sedghi et al. (2002); Sedghi (2003) is an equation-based representation.

In the next section, $H_{\mathrm{DDS}_{1}}$ is rewritten for stick-slip systems with discontinuous friction. In Section 4 , a variation of $H_{\mathrm{DDS}_{1}}$ is considered for stick-slip systems. It includes transition states between stick and slip phases in order to take into account friction models which are more appropriate for simulation purposes. This extended DDS hybrid automaton is valid for DDS with one discontinuity surface, although for space reasons, it is only presented for systems with discontinuous friction.

\section{THE DDS HYBRID AUTOMATON FOR SYSTEMS WITH FRICTION AND STICK-SLIP}

The DDS hybrid automaton $H_{\mathrm{DDS}_{1}}$ is rewritten for a system with discontinuous friction: a simplified 2-DOF-model of a vertical oilwell drillstring. It is a particular case of the $n$ DOF model proposed in Navarro-López and Cortés (2007). The change in the number of DOF's only implies the change in the dimension of the continuous state space within each location.

The drillstring torsional behaviour is described by a simple torsional pendulum driven by an electrical motor, and the bitrock contact is described by a dry friction model. The drill pipes are represented by a linear spring with torsional stiffness $k_{\mathrm{t}}$ and a torsional damping $c_{\mathrm{t}}$, which connect the inertias $J_{\mathrm{r}}$ and $J_{\mathrm{b}}$.

The system state vector is $\mathbf{x}=\left(\dot{\varphi}_{\mathrm{r}}, \varphi_{\mathrm{r}}-\varphi_{\mathrm{b}}, \dot{\varphi}_{\mathrm{b}}\right)^{\mathrm{T}}$, with $\varphi_{\mathrm{i}}, \dot{\varphi}_{\mathrm{i}}$ $(i \in\{r, b\})$ the angular displacements and angular velocities of the top-rotary system and the bit. At the top-drive system, a viscous damping torque is considered $\left(c_{\mathrm{r}} x_{1}\right) . T_{\mathrm{m}}=u$ is the torque applied by a motor at the surface and is considered as a constant input. $T_{\mathrm{b}}\left(x_{3}\right)=c_{\mathrm{b}} x_{3}+T_{\mathrm{f}_{\mathrm{b}}}\left(x_{3}\right)$ is the torque on the bit with $c_{\mathrm{b}} x_{3}$ approximating the influence of the mud drilling on the bit behaviour. $T_{\mathrm{f}_{\mathrm{b}}}\left(x_{3}\right)$ is the friction modelling the bit-rock contact, and $T_{\mathrm{f}_{\mathrm{b}}}\left(x_{3}\right)=f_{\mathrm{b}}\left(x_{3}\right) \operatorname{sign}\left(x_{3}\right)$ with:

$$
f_{\mathrm{b}}\left(x_{3}\right)=W_{\mathrm{ob}} R_{\mathrm{b}}\left[\mu_{\mathrm{c}_{\mathrm{b}}}+\left(\mu_{\mathrm{s}_{\mathrm{b}}}-\mu_{\mathrm{c}_{\mathrm{b}}}\right) \exp ^{-\frac{\gamma_{\mathrm{b}}}{v_{\mathrm{f}}}\left|x_{3}\right|}\right] \text {, }
$$

with $W_{\mathrm{ob}}>0$ the weight on the bit (considered as a constant input), $R_{\mathrm{b}}>0$ the bit radius; $\mu_{\mathrm{s}_{\mathrm{b}}}, \mu_{\mathrm{c}_{\mathrm{b}}} \in(0,1)$ the static and Coulomb friction coefficients associated with $J_{\mathrm{b}}, 0<\gamma_{\mathrm{b}}<1$ and $v_{\mathrm{f}}>0$. In addition, the Coulomb and static friction torque is $T_{\mathrm{c}_{\mathrm{b}}}$ and $T_{\mathrm{s}_{\mathrm{b}}}$, respectively, with $T_{\mathrm{c}_{\mathrm{b}}}=W_{\mathrm{ob}} R_{\mathrm{b}} \mu_{\mathrm{c}_{\mathrm{b}}}, T_{\mathrm{s}_{\mathrm{b}}}=W_{\mathrm{ob}} R_{\mathrm{b}} \mu_{\mathrm{s}_{\mathrm{b}}}$. The sign function is considered as:

$$
\operatorname{sign}\left(x_{3}\right)=x_{3} /\left|x_{3}\right| \text { if } x_{3} \neq 0, \operatorname{sign}\left(x_{3}\right) \in[-1,1] \text { if } x_{3}=0 .
$$

The drillstring dynamics is given by:

$$
\begin{aligned}
& \dot{x}_{1}=\frac{1}{J_{\mathrm{r}}}\left[-\left(c_{\mathrm{t}}+c_{\mathrm{r}}\right) x_{1}-k_{\mathrm{t}} x_{2}+c_{\mathrm{t}} x_{3}+u\right], \dot{x}_{2}=x_{1}-x_{3}, \\
& \dot{x}_{3}=\frac{1}{J_{\mathrm{b}}}\left[c_{\mathrm{t}} x_{1}+k_{\mathrm{t}} x_{2}-\left(c_{\mathrm{t}}+c_{\mathrm{b}}\right) x_{3}-T_{\mathrm{f}_{\mathrm{b}}}\left(x_{3}\right)\right],
\end{aligned}
$$

or in a compact form, $\dot{\mathbf{x}}(t)=\mathbf{A x}(t)+\mathbf{B} u(t)+\mathbf{T}_{\mathrm{f}}(\mathbf{x}(t))$, where $\mathbf{A}, \mathbf{B}$ are constant matrices and $\mathbf{T}_{\mathbf{f}}$ represents the torque on the bit. The inputs of the system are $u$ and $W_{\mathrm{ob}}$. The outputs are the angular velocities $x_{1}$ and $x_{3}$.

For (7) with (5), $s(\mathbf{x})=x_{3}$, the switching surface is $S=\{\mathbf{x} \in$ $\left.\mathbb{R}^{3}: x_{3}=0\right\}$, and the sliding set is $S_{\mathrm{s}}=\left\{\mathbf{x} \in S: \mid c_{\mathrm{t}} x_{1}+\right.$ $\left.k_{\mathrm{t}} x_{2} \mid \leq T_{\mathrm{s}_{\mathrm{b}}}\right\}$. Notice that $T_{\mathrm{f}_{\mathrm{b}}}$ plays the role of the equivalent control $\left(T_{\text {fbeq }}\right)$, and $T_{\text {fbeq }}$ is the solution for $T_{\mathrm{f}_{\mathrm{b}}}$ of equation $\dot{s}=0$, that is, $u_{\mathrm{eq}}=T_{\text {fbeq }}=c_{\mathrm{t}} x_{1}+k_{\mathrm{t}} x_{2}-\left(c_{\mathrm{t}}+c_{\mathrm{b}}\right) x_{3}$. Moreover, $-T_{\mathrm{s}_{\mathrm{b}}} \leq T_{\mathrm{fbeq}} \leq T_{\mathrm{s}_{\mathrm{b}}}$. For more details, the reader is invited to read Navarro-López and Cortés (2007); Navarro-López and Licéaga-Castro (2009a); Navarro-López (2009b).

The DDS hybrid automaton $H_{\mathrm{DDS}_{1}}$ associated with system (7)(5) has the following vector fields and guard sets:

$$
\begin{aligned}
& \mathbf{f}_{\mathrm{q} 1}\left(\mathbf{x}, W_{\mathrm{ob}}, u\right)=\mathbf{A} \mathbf{x}+\mathbf{B} u+\left.\mathbf{T}_{\mathrm{f}}(\mathbf{x})\right|_{T_{\mathrm{f}_{\mathrm{b}}}=T_{\mathrm{f}_{\mathrm{b}}}^{+}}=f^{+}(\mathbf{x}), \\
& \mathbf{f}_{\mathrm{q} 2}\left(\mathbf{x}, W_{\mathrm{ob}}, u\right)=\mathbf{A} \mathbf{x}+\mathbf{B} u+\left.\mathbf{T}_{\mathrm{f}}(\mathbf{x})\right|_{T_{\mathrm{f}_{\mathrm{b}}}=T_{\mathrm{f}_{\mathrm{b}}}}=f^{-}(\mathbf{x}), \\
& \mathbf{f}_{\mathrm{q} 3}(\mathbf{x}, u)=\left(\begin{array}{c}
\frac{1}{J_{\mathrm{r}}}\left[-\left(c_{\mathrm{t}}+c_{\mathrm{r}}\right) x_{1}-k_{\mathrm{t}} x_{2}+u\right] \\
x_{1} \\
0
\end{array}\right)=f_{\mathrm{s}}(\mathbf{x}), \\
& G\left(q_{1}, q_{3}\right)=G\left(q_{2}, q_{3}\right)=\left\{\mathbf{x} \in S:\left|c_{\mathrm{t}} x_{1}+k_{\mathrm{t}} x_{2}\right| \leq T_{\mathrm{s}_{\mathrm{b}}}\right\}, \\
& G\left(q_{1}, q_{2}\right)=G\left(q_{3}, q_{2}\right)=\left\{\mathbf{x} \in S: c_{\mathrm{t}} x_{1}+k_{\mathrm{t}} x_{2}<-T_{\mathrm{s}_{\mathrm{b}}}\right\}, \\
& G\left(q_{2}, q_{1}\right)=G\left(q_{3}, q_{1}\right)=\left\{\mathbf{x} \in S: c_{\mathrm{t}} x_{1}+k_{\mathrm{t}} x_{2}>T_{\mathrm{s}_{\mathrm{b}}}\right\},
\end{aligned}
$$

where $T_{\mathrm{f}_{\mathrm{b}}}^{+}$and $T_{\mathrm{f}_{\mathrm{b}}}^{-}$are $T_{\mathrm{f}_{\mathrm{b}}}\left(x_{3}\right)$ for $x_{3}>0$ and $x_{3}<0$, respectively. The directed graph associated with this DDS hybrid automaton is shown in Fig. 1. The guards are close to the departure locations, and the reset functions are close to the arrival locations.

The 3-discrete-states hybrid automaton proposed here overcomes some non-determinism problems encountered in other hybrid formulations of discontinuous systems with sliding, for example, like the one presented in Mattsson (1996) where an object-oriented model with 3 discrete locations is used.

\section{MODIFYING THE DDS HYBRID AUTOMATON}

A new hybrid automaton with five discrete locations is proposed. It will be called the extended DDS hybrid automaton $H_{\mathrm{DDS}_{2}}$, and is inspired in the simulation-oriented friction models which include intermediate transitions when leaving the discontinuity surface (stick-to-slip transition). This new model is of general purpose, although it is only presented for systems with discontinuous friction.

For the example of Section 3, the discontinuous element is the friction, which is now considered as a combination of the switch 


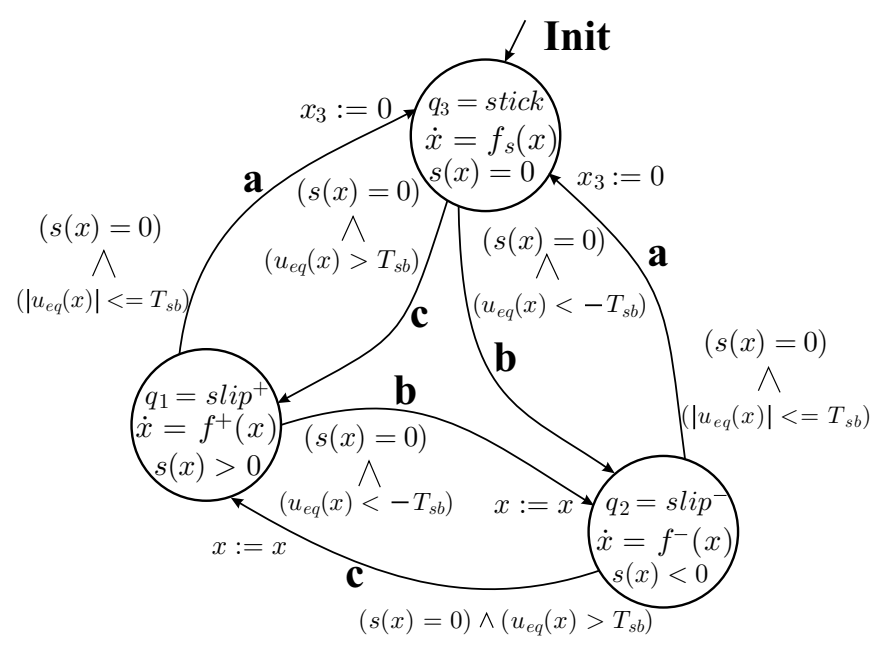

Fig. 1. Directed graph associated with the DDS hybrid automaton $H_{\mathrm{DDS}_{1}}$ for the drillstring with $x_{0}=\left(x_{1}, x_{2}, 0\right)^{\mathrm{T}}$.

model Leine et al. (1998) and the Karnopp's model, in which a zero velocity band is introduced Karnopp (1985). Thus,

$$
T_{\mathrm{f}_{\mathrm{b}}}(\mathbf{x})= \begin{cases}T_{\mathrm{e}_{\mathrm{b}}}(\mathbf{x}) & \text { if }\left|x_{3}\right| \leq \delta,\left|T_{\mathrm{e}_{\mathrm{b}}}\right| \leq T_{\mathrm{s}_{\mathrm{b}}}(\text { stick }), \\ T_{\mathrm{s}_{\mathrm{b}}} \operatorname{sign}\left(T_{\mathrm{e}_{\mathrm{b}}}(\mathbf{x})\right) & \text { if }\left|x_{3}\right| \leq \delta,\left|T_{\mathrm{e}_{\mathrm{b}}}\right|>T_{\mathrm{S}_{\mathrm{b}}} \\ & (\text { stick-to-slip transition) } \\ f_{\mathrm{b}}\left(x_{3}\right) \operatorname{sign}\left(x_{3}\right) & \text { if }\left|x_{3}\right|>\delta \text { (sliding) }\end{cases}
$$

where $\delta>0$, and $T_{\mathrm{e}_{\mathrm{b}}}$ is the reaction torque, which coincides with the equivalent control. Then the DDS hybrid automaton $H_{\mathrm{DDS}_{1}}$ is modified in such a manner to obtain the extended DDS hybrid automaton $H_{\mathrm{DDS}_{2}}$. For limited space reasons, the discrete states, transitions, guard and location domain sets, and reset functions are only shown in the graphical representation of the hybrid dynamical system of Fig. $2 . \dot{s}$ denotes the time derivative of function $s$ along the system trajectories.

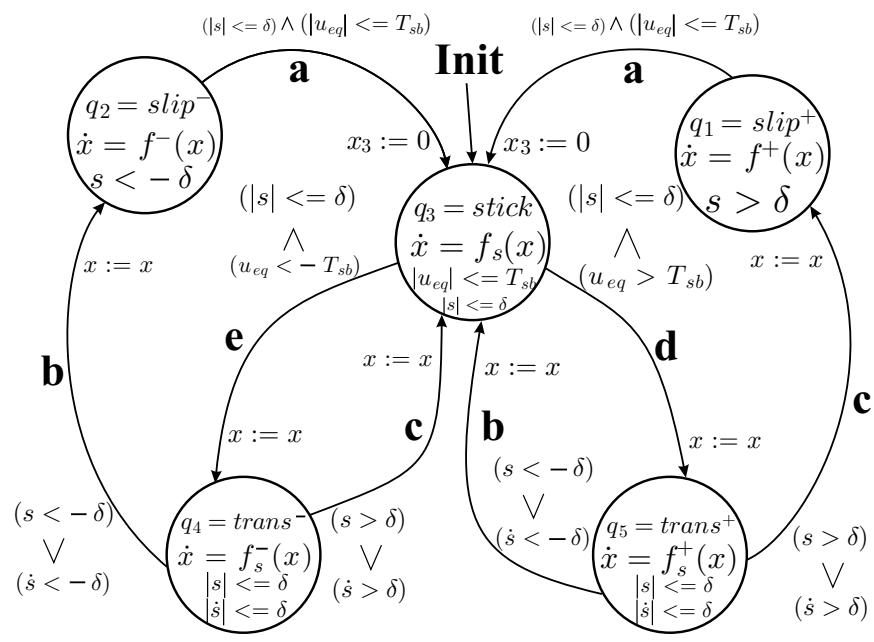

Fig. 2. Directed graph associated with the extended DDS hybrid automaton $H_{\mathrm{DDS}_{2}}$ for the drillstring with $x_{0}=\left(x_{1}, x_{2}, 0\right)^{\mathrm{T}}$.

$H_{\mathrm{DDS}_{1}}$ has been modified in order to include two intermediate states before motion (one for positive velocities, another for negative velocities), such as: $q_{5}=\left\{\right.$ trans $\left.^{+}\right\}$and $q_{4}=\left\{\right.$ trans $\left.^{-}\right\}$. Indeed, what we are doing is splitting the stick state into two parts: first, when the trajectories are within the sliding set $\left(q_{3}\right)$, second, when the trajectories are within the crossing set on the discontinuity surface $\left(q_{4}\right.$ or $\left.q_{5}\right)$. The necessity of these states for simulation purposes is well-known, see for example Elmqvist et al. (1993) and Mattsson (1996). The extended DDS hybrid automaton $H_{\mathrm{DDS}_{2}}$ is also inspired in the state-transition diagram of a friction model presented in Elmqvist et al. (1993). The vector fields associated with $q_{4}\left(f_{\mathrm{s}}^{-}(\mathbf{x})\right)$ and $q_{5}\left(f_{\mathrm{s}}^{+}(\mathbf{x})\right)$ have to be different than the vector field within $q_{3}$. They are obtained by considering in (7), $x_{3}=0$ and:

$$
T_{\mathrm{f}_{\mathrm{b}}}=\max \left(u_{\mathrm{eq}}\right) \operatorname{sign}\left(u_{\mathrm{eq}}\right)=T_{\mathrm{s}_{\mathrm{b}}} \operatorname{sign}\left(T_{\mathrm{e}_{\mathrm{b}}}\right) \text {. }
$$

Furthermore, there is no direct switching between slip ${ }^{+}$and slip $^{-}$or viceversa. For example, if a transition slip ${ }^{+} \rightarrow$ slip $^{-}$ should be carried out, if the system is in state slip ${ }^{+}$and $s$ becomes zero, the system switches to stick before checking the conditions for switching to slip ${ }^{-}$. Moreover, if the system is in location trans $^{+}$and the velocity is reversed before starting to move, the system switches to state stick before going to slip $^{-}$. This is a way to avoid the non-determinism discussed in Mattsson (1996).

\section{SIMULATION OF THE HYBRID AUTOMATA}

Stateflow ${ }^{\circledR}$ under Simulink ${ }^{\circledR}$ in MATLAB ${ }^{\circledR}$ The MathWorks, Inc. (1999-2003) is the software package used to simulate the two DDS hybrid automata.

Although the translation of the hybrid automata $H_{\mathrm{DDS}_{1}}$ and $H_{\mathrm{DDS}_{2}}$ into Stateflow charts is almost immediate, some important aspects have to be pointed out:

- The location domain sets in each discrete state are translated into self-loop transitions in the Stateflow chart. This becomes more critical in $H_{\mathrm{DDS}_{2}}$, since more variables and conditions are associated with the transitions or events. In $H_{\mathrm{DDS}_{1}}$, the same result is obtained whether or not self-loop transitions are included, due to the fact that the guard sets uniquely define the switchings between discrete states.

- Due to the difficulty in detecting the zero-crossing of the functions involved, in $H_{\mathrm{DDS}_{1}}, s=0$ is checked by means of the condition $s_{z} z$ ero $==1$. The boolean variable $s \_z e r o$ is obtained by passing $s=x_{3}$ through the Threshold Simulink block, and it is an external input to the Stateflow chart.

The Stateflow charts designed are not included for limited space reasons, and they look exactly the same (for $H_{\mathrm{DDS}_{2}}$, with additional self-loop discrete-state transitions) as the directed graphs associated with $H_{\mathrm{DDS}_{1}}$ and $H_{\mathrm{DDS}_{2}}$ given in Figs. 1 and 2 .

The simulation results for the two hybrid automata are shown in Figs. 3, 4, 5, 6 and 7. The hybrid systems simulation under Stateflow ${ }^{R}$ is compared with the simulation of the discontinuous system (7) with the friction model (9) (in this case, the integration function used is ode 45 of MATLAB ${ }^{\mathrm{R}}$ ). The parameters used for the simulations are:

$$
\begin{aligned}
& J_{r}=2122 \mathrm{~kg} \mathrm{~m}^{2}, J_{b}=471.9698 \mathrm{~kg} \mathrm{~m}^{2}, R_{b}=0.155575 \mathrm{~m}, \\
& k_{t}=698.063 \mathrm{Nm} / \mathrm{rad}, c_{t}=139,6126 \mathrm{Nm} / \mathrm{rad}, \\
& c_{r}=425 \mathrm{Nms} / \mathrm{rad}, c_{b}=50 \mathrm{Nms} / \mathrm{rad}, \mu_{c_{b}}=0.5, \\
& \mu_{s_{b}}=0.8, \delta=10^{-3}, \gamma_{b}=0.9, v_{f}=1 .
\end{aligned}
$$

From the figures, it can be seen how the hybrid automata reproduce the three main types of bit dynamical behaviours, 
such as: 1) stick-slip at $x_{3}$, that is, the trajectory enters and leaves repeatedly the sliding mode; 2 ) permanent stuck bit, i.e., $x(t) \in S, \forall t>t_{\mathrm{s}}$, for some $\left.t_{\mathrm{s}}>0 ; 3\right)$ the bit moves with a positive constant velocity.

Several features are observed from the simulations. First of all, the importance of the step size of the numerical integration method. For simulating the hybrid models, the function ode 45 of MATLAB ${ }^{\mathrm{R}}$ is used with a variable-step size integration method. The maximum step size is changed in order to appreciate its effect on the system solutions obtained.

In Figs. 3, 4 and 5, the maximum step size is $h=0.001 \mathrm{sec}$. With this $h$, the trajectories of the three systems coincide for the three dynamical behaviours. Differences in the dynamics appear when $h$ is not small enough. In addition, the bigger the number of entries into the discontinuity surface is, that is, the bigger the number of transitions between discrete locations is, the bigger the difference between the two hybrid trajectories given by $H_{\mathrm{DDS}_{1}}$ and $H_{\mathrm{DDS}}$ is, and the bigger the difference between the hybrid trajectories and the trajectory of system (7)(9) is. See Figs. 6 and 7, where a maximum $h=2 \mathrm{sec}$ was used. This fact is specially visible in Fig. 6.(1) and 7.

Hence, the number of discrete locations and transitions in a hybrid automaton can change the system behaviour. This is, mainly, because there are two phenomena associated with every event $(E)$. There is an instantaneous transition in the discrete state and another in the continuous part of the state, that is: a switch (transition, guard) and a jump (reset). It has to be also pointed out that in the evolution of $q$ in $H_{\mathrm{DDS}_{2}}$, the change from $q=3$ to $q=5$ is of impulsive type. The reason is of numerical type. When $q=3$ and $u_{\text {eq }}$ becomes greater than $T_{\mathrm{s}_{\mathrm{b}}}$, there is a delay so that this change in $u_{\mathrm{eq}}$ can be detected.

To conclude with, there are changes introduced in the system dynamical behaviour by the hybrid-automaton representation. Numerical aspects have to be also taken into account.

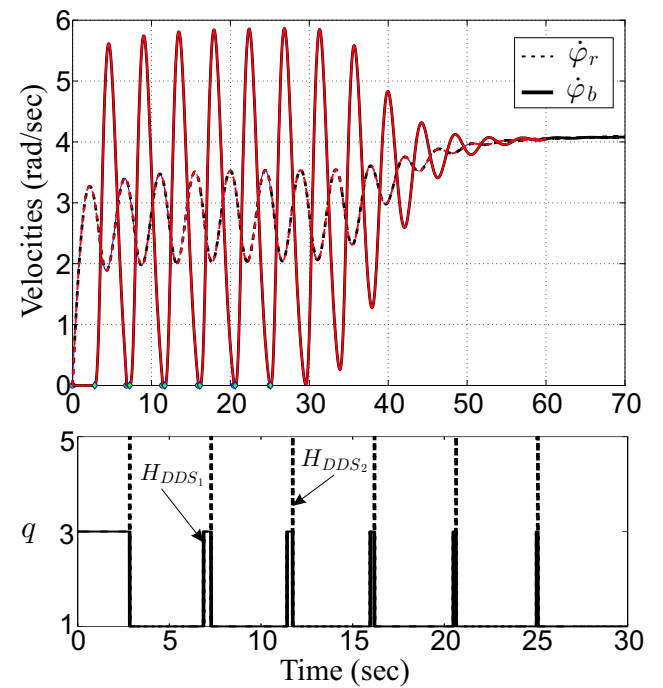

Fig. 3. Convergence to the equilibrium entering several times the switching surface for the three systems: $u=6 \mathrm{kNm}$, $W_{o b}=51408 \mathrm{~N}$. A small enough step size is used.

\section{CONCLUSION}

Two hybrid-automaton-based models have been compared for the specification of discontinuous systems exhibiting sliding-
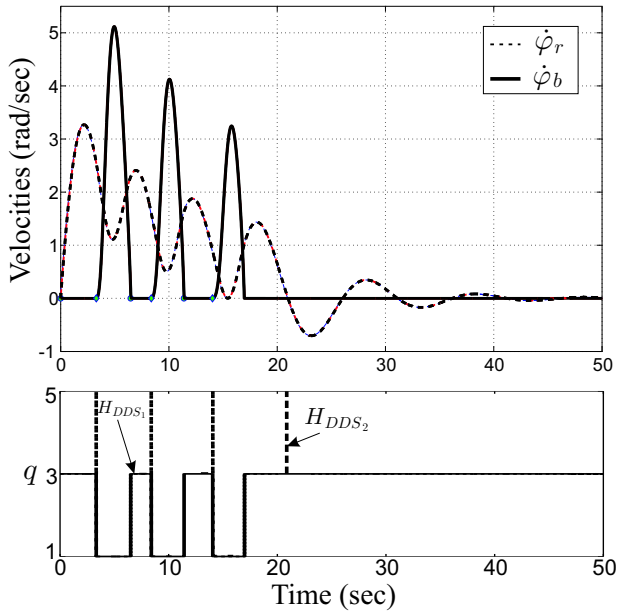

Fig. 4. Permanent stuck bit for the three systems: $u=6 \mathrm{kNm}$, $W_{o b}=60 \mathrm{kN}$. A small enough step size is used.

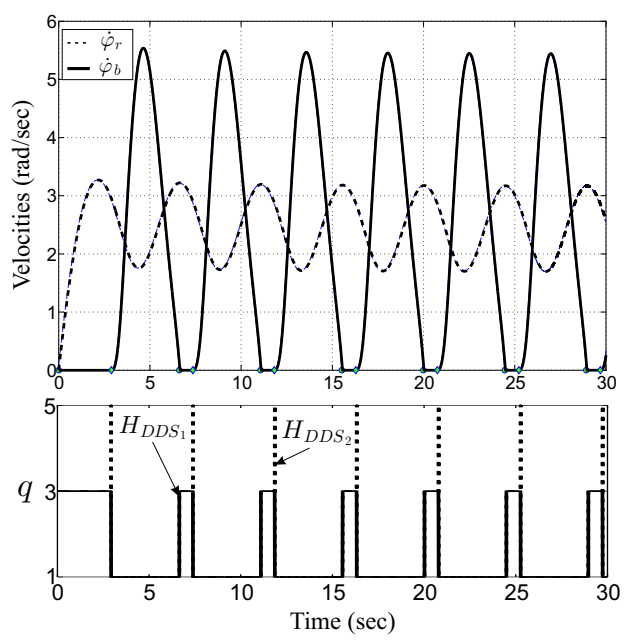

Fig. 5. Stick-slip situation for the three systems: $u=6 \mathrm{kNm}$, $W_{o b}=53018 \mathrm{~N}$. A small enough step size is used.
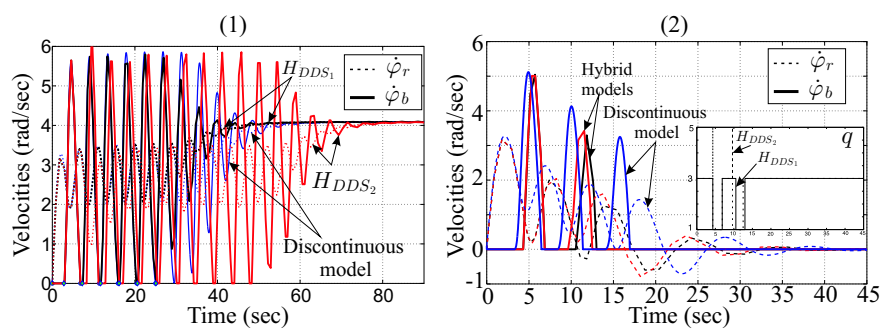

Fig. 6. Use of a bigger step size in the numerical integration method: (1) Convergence to the equilibrium: $u=6 \mathrm{kNm}$, $W_{o b}=51408 \mathrm{kN}$, (2) permanent stuck bit: $u=6 \mathrm{kNm}$, $W_{o b}=60 \mathrm{kN}$.

mode behaviour. An example has been used to illustrate the models. It is a simplified torsional model of a drillstring with discontinuous friction and stick-slip. Under the framework proposed, the complex behaviours associated with discontinuous dynamical systems can be reinterpreted and redefined from a computational viewpoint. However, this paper poses more questions than answers, which must be taken into account when hybrid models are considered for modelling physical systems. 

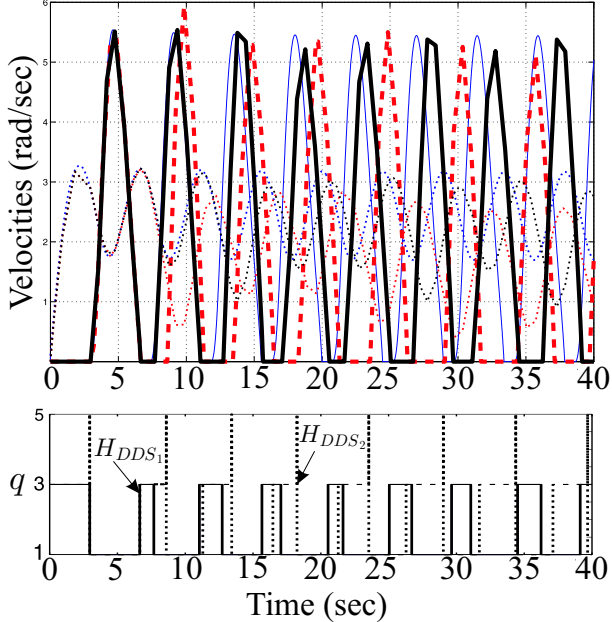

Fig. 7. Use of a bigger step size in the numerical integration method: Stick-slip situation with $u=6 \mathrm{kNm}, W_{o b}=$ $53018 \mathrm{~N}$. $-\dot{\varphi}_{\mathrm{b}}$ obtained with $H_{\mathrm{DDS}_{1}},=-\dot{\varphi}_{\mathrm{b}}$ obtained with $H_{\mathrm{DDS}_{2}},-\dot{\varphi}_{\mathrm{b}}$ of system (7)-(9), $\cdots \dot{\varphi}_{\mathrm{r}}$.

\section{ACKNOWLEDGEMENTS}

Thank you very much to Rebekah Carter for her comments.

\section{REFERENCES}

The MathWorks, Inc. Stateflow and Stateflow Coder User's Guide. For Complex Logic and State Diagram Modeling. http: / / www . mathworks. com/access/helpdesk _r13/help/pdf_doc/stateflow/sf_ug.pdf.

V. Acary, and B.Brogliato. Numerical Methods for Nonsmooth Dynamical Systems. Springer Verlag LNACM, 35, Heidelberg, 2008.

A. Agrawal, G. Simon, and G. Karsai. Semantic translation of Simulink/Stateflow models to hybrid automata using graph transformations. Electronic Notes in Theoretical Computer Science, 109:43-56, 2004.

R. Alur, A. Kanade, S. Ramesh, and K.C. Shashidhar. Symbolic analysis for improving simulation coverage of Simulink/Stateflow models. 8th ACM and IEEE International Conference on Embedded software, EMSOFT 2008, (Atlanta, USA, October 19-24), 2008.

R. Alur, C. Courcoubetis, T.A. Henzinger, and P.H. Ho. Hybrid automata: An algorithmic approach to the specification and verification of hybrid systems. Lecture Notes in Computer Science, 736:209-229, 1993.

B. Brogliato. Nonsmooth Mechanics. Springer, London, 2nd ed., 1999.

M. Buss, M. Glocker, M. Hardt, O. von Stryk, R. Bulirsch, and G. Schmidt. Nonlinear hybrid dynamical systems: Modelling, optimal control, and applications. Modelling, Analysis and Design of Hybrid Systems, LNCIS 279:311335, Springer-Verlag, Berlin, 2002.

H. Elmqvist, F.E. Cellier, and M. Otter. Object-oriented modeling of hybrid systems. European Simulation Symposium, (Delft, The Netherlands)31-41, 1993.

A.F. Filippov. Differential Equations with Discontinuous Righthand Sides. Kluwer Academic Publishers, Dordrecht, 1988.

T.A. Henzinger. The theory of hybrid automata. Proc. 11th IEEE Symposium of Logic in Computer Science, 1:278-292, 1996.
K.H. Johansson, M. Egerstedt, J. Lygeros, and S. Sastry. On the regularization of Zeno hybrid automata. Systems \& Control Letters, 38:141-150, 1999.

D. Karnopp. Computer simulation of stick-slip friction in mechanical dynamic systems. ASME Journal of Dynamic Systems, Measurement, and Control, 107:100-103, 1985.

Y.A. Kuznetsov, S. Rinaldi, and A. Gragnani. One-parameter bifurcations in planar Filippov systems. International Journal of Bifurcations and Chaos, 13(8):2157-2188, 2003.

R.I. Leine, D.H. van Campen, A. de Kraker, and L. van den Steen. StickÜslip vibrations induced by alternate friction models. Nonlinear Dynamics, 16:41-Ü54, 1998.

P. Lötstedt. Coulomb friction in two-dimensional rigid body systems. Z. Angew. Math. und Mech., 64:605-615, 1981.

J. Lygeros, C. Tomlin, and S. Sastry. Controllers for reachability specifications for hybrid systems. Automatica, 35(3): 349-370, 1999.

J. Lygeros, K.H. Johansson, S.N. Simić, J. Zhang, and S. Sastry. Dynamical properties of hybrid automata. IEEE Transactions on Automatic Control, 48(1):2-17, 2003.

S.E. Mattsson. On object-oriented modelling of relays and sliding mode behaviour. 13th Triennial IFAC World Congress, (San Francisco, USA), 1996, pp. 259-264.

P.J. Mosterman, F. Zhao, and G. Biswas. Sliding mode model semantics and simulation for hybrid systems. P. Antsaklis et al. (Eds); Hybrid Systems V, LNCS, 1567:218-237, SpringerVerlag, 1999.

P.J. Mosterman, and G. Biswas. A comprehensive methodology for building hybrid models of physical systems. Artificial Intelligence, 121:171-209, 2000.

E.M. Navarro-López, and D. Cortés. Avoiding harmful oscillations in a drillstring through dynamical analysis. Journal of Sound and Vibration, 307(1-2):152-171, 2007.

E.M. Navarro-López and E. Licéaga-Castro. Non-desired transitions and sliding-mode control of a multi-DOF mechanical system with stick-slip oscillations. Chaos, Solitons \& Fractals, 41:2035-2044, 2009.

E.M. Navarro-López. An alternative characterization of bitsticking phenomena in a multi-degree-of-freedom controlled drillstring. Nonlinear Analysis: Real World Applications, 10: 3162-3174, 2009.

E.M. Navarro-López. Hybrid modelling of a discontinuous dynamical system including switching control. 2nd IFAC Conference on Analysis and Control of Chaotic Systems, (London, UK, June 22-24), 2009.

T. Park, and P.I. Barton. State event location in differentialalgebraic models. ACM Transactions on Modeling and Computer Simulation, 6(2):137-165, 1996.

B. Sedghi, B. Srinivasan, and R. Longchamp. Control of hybrid systems via dehybridization. American Control Conference, (Anchorage, Alaska, USA, May 8-10), 2002.

B. Sedghi. Control Design of Hybrid Systems via Dehybridization. $\mathrm{PhD}$ thesis, Ecole Polytechnique Fédérale de Lausanne, Lausanne, Switzerland, 2003.

V.I. Utkin. Sliding Modes in Control Optimization. SpringerVerlag, Berlin, 1992.

F. Zhang, M. Yeddanapudi, and P.J. Mosterman. Zero-crossing location and detection algorithms for hybrid system simulation. 17th IFAC Triennial World Congress, (Seoul, Korea, July 6-11)7967-7972, 2008.

F. Zhao, and V.I. Utkin. Adaptive simulation and control variable-structure control systems in sliding regimes. Automatica, 32(7):1037-1042, 1996. 